\title{
Nrf2 activation induces mitophagy and reverses Parkin/Pink1 knock down-mediated neuronal and muscle degeneration phenotypes
}

\author{
Sentiljana Gumeni ${ }^{1}$, Eleni-Dimitra Papanagnou ${ }^{1}$, Maria S. Manola (D) $^{1}$ and Ioannis P. Trougakos ${ }^{1 凶}$
}

(c) The Author(s) 2021

The balanced functionality of cellular proteostatic modules is central to both proteome stability and mitochondrial physiology; thus, the age-related decline of proteostasis also triggers mitochondrial dysfunction, which marks multiple degenerative disorders. Nonfunctional mitochondria are removed by mitophagy, including Parkin/Pink1-mediated mitophagy. A common feature of neuronal or muscle degenerative diseases, is the accumulation of damaged mitochondria due to disrupted mitophagy rates. Here, we exploit Drosophila as a model organism to investigate the functional role of Parkin/Pink1 in regulating mitophagy and proteostatic responses, as well as in suppressing degenerative phenotypes at the whole organism level. We found that Parkin or Pink1 knock down in young flies modulated proteostatic components in a tissue-dependent manner, increased cell oxidative load, and suppressed mitophagy in neuronal and muscle tissues, causing mitochondrial aggregation and neuromuscular degeneration. Concomitant to Parkin or Pink1 knock down cncC/Nrf2 overexpression, induced the proteostasis network, suppressed oxidative stress, restored mitochondrial function, and elevated mitophagy rates in flies' tissues; it also, largely rescued Parkin or Pink1 knock downmediated neuromuscular degenerative phenotypes. Our in vivo findings highlight the critical role of the Parkin/Pink1 pathway in mitophagy, and support the therapeutic potency of Nrf2 (a druggable pathway) activation in age-related degenerative diseases.

Cell Death and Disease (2021)12:671; https://doi.org/10.1038/s41419-021-03952-w

\section{INTRODUCTION}

Mitochondria are very dynamic organelles as they change their shape, size, and sub-cellular localization depending on the versatile cellular demands for adequate ATP production, optimal cellular function, and survival [1]. A common feature of neurodegenerative conditions such as Parkinson's (PD) and Alzheimer's (AD) disease is mitochondria dysfunction [2,3]. PD is the second most common neurodegenerative disease, characterized by loss of dopaminergic (DA) neurons, rigidity, bradykinesia, postural instability, and tremor [4].

The maintenance of cellular mitostasis directly correlates with mitochondria quality control, which is driven by mitochondrial dynamics, mitochondrial removal, and biogenesis. A terminal response to mitochondria dysfunction is their selective destruction by mitophagy [5]; a highly conserved process, where damaged mitochondria are removed by autophagosomes and delivered for degradation to lysosomes [6]. One of the main pathways involved in this process is the Parkin/PTEN-induced putative kinase 1 (Pink1)-mediated mitophagy [6]. Pink1 is activated by auto-phosphorylation and accumulates on the outer mitochondrial membrane (OMM) of dysfunctional mitochondria triggering the recruitment of the E3 ubiquitin ligase Parkin, which then ubiquitinates several proteins of OMM, amplifying a cascade signal that culminates in mitochondrial engulfment by the autophagosome [7-9]. Parkin and Pink1 are the two most commonly mutated proteins in autosomal-recessive juvenile Parkinsonism $[10,11]$. Mitophagy disruption is also a hallmark of $A D$ [12], further supporting the importance of mitochondria quality control in neurodegeneration.

Reactive oxygen species (ROS) are main by-products of mitochondria functionality [13] and elevated intracellular ROS levels can cause damage to all cellular biomolecules [14]. A master regulator of cellular redox, and also of proteome homeostasis (referred to as, proteostasis), is the nuclear factor erythroid 2 like 2 [Nrf2; a cap " $\mathrm{n}$ " collar (cnc)] transcription factor [15]. Key components of the proteostatic pathways are the molecular chaperones and the two main degradation machineries, namely the autophagy lysosome-(ALP) and the ubiquitin proteasome(UPP) pathways; ALP is mostly involved in the degradation of protein aggregates and damaged organelles [8], while UPP ensures protein synthesis quality control and degradation of short-lived regulatory proteins [16]. The 26S eukaryotic proteasome comprises a 205 core particle being bound to 195 regulatory particles. The $20 \mathrm{~S}$ proteasome consists of four stacked heptameric rings (two a-type surrounding two $\beta$-type) with the caspase-like $(\mathrm{C}-\mathrm{L})$, trypsin-like (T-L), and chymotrypsin-like (CT-L) proteasome enzymatic activities located at the $\beta 1, \beta 2$, and $\beta 5$ subunits, respectively [17]. Reportedly, proteasomal degradation is particularly important in PD as it degrades insoluble protein inclusions of $a$-synuclein [18]. Proteasome and mitochondria are highly

${ }^{1}$ Department of Cell Biology and Biophysics, Faculty of Biology, National and Kapodistrian University of Athens, Athens 15784, Greece. ${ }^{\otimes}$ email: itrougakos@biol.uoa.gr. Edited by M Agostini

Received: 11 January 2021 Revised: 26 May 2021 Accepted: 26 May 2021

Published online: 03 July 2021 
interdependent, since UPP requires excessive amounts of ATP and the ubiquitinated OMM proteins are degraded by the proteasome [1]. Notably, the declined functionality of UPP and ALP is a hallmark of aging and of several age-related diseases, including neuromuscular degeneration [19].

Additionally, to its role in redox and proteome homeostasis, Nrf2 also affects several aspects of mitostasis, including mitochondrial biogenesis, fatty acid oxidation, respiration, ATP production, and the structural integrity of the organelle [20]. Our in vivo studies in the fly model have revealed that the Nrf2 Drosophila ortholog, namely cncC (hereafter referred to as cncC/Nrf2), regulates mitochondria biogenesis [21], while disruption of mitochondria dynamics triggers a $\mathrm{cncC} / \mathrm{Nrf2}$-dependent proteostatic response [22]. In C. elegans, Nrf2 becomes activated upon inhibition of mitophagy to promote the expression of mitochondrial biogenesis and mitophagy genes [23]; in support, the mitophagic/autophagic adapter protein sequestosome-1 (SQSTM1/p62) is a Nrf2 transcriptional target [23-25].

Herein, we exploit Drosophila as a model organism to investigate the functional role of Parkin/Pink1 in regulating mitophagy and proteostatic responses, as well as in suppressing degeneration-like phenotypes at the whole organism level. We found that Parkin (known as park in Drosophila) or Pink1 knock down (KD) increased cell oxidative load and suppressed mitophagy causing neuronal and muscle degeneration. Concomitant to park or Pink1 KD activation of $\mathrm{cncC} / \mathrm{Nrf2}$, induced the proteostasis network, suppressed oxidative stress, restored (independently to $\operatorname{ref}(2) \mathrm{P} / \mathrm{p} 62$ ) mitophagy and largely rescued park or Pink1 KD-mediated neuromuscular degenerative phenotypes.

\section{MATERIALS AND METHODS \\ Flies' culture and stocks}

Flies' stocks were maintained at $25^{\circ} \mathrm{C}, 60 \%$ relative humidity on a $12 \mathrm{~h}$ light: $12 \mathrm{~h}$ dark cycle and were fed standard medium (unless otherwise indicated). The transgenic strains $\mathrm{w}^{1118}$ (\#5905), UAS Pink1 RNAi (\#55886), UAS Pink1 (\#51648), UAS park RNAi (\#38333), UAS park (\#51651), UAS ref(2) P RNAi (\#36111), Gal4-Mef2 (\#27390), UAS Mito-GFP (\#8443), tub-mitoroGFP2-Orp1 (\#67673); the nervous system specific Gal4-Elav (\#8765) and the dopaminergic neurons Gal4-TH (\#8848) were obtained from the Bloomington Stock Center. The double transgenic line UAS Lamp1-GFP, Actin Gal4 was kindly donated by Dr. G. Orso (University of Padova). The UAS cncC/Nrf2, UAS cncC/Nrf2 RNAi and the Tubulin GeneSwitch Gal4 (tubGSGal4) flies were a gift from Prof. D. Bohmann (University of Rochester, NY, USA); the conditional driver tubGSGal4 is ubiquitously activated upon dietary administration of RU486 $(320 \mu \mathrm{M})$. Mito-QC was kindly donated by Prof. A. Whitworth (University of Cambridge, UK). Given that gonads display distinct aging rates and regulation of proteostatic mechanisms as compared to adult somatic tissues [26], in all presented experiments (unless otherwise indicated) referring to adult flies only microdissected somatic tissues (head and thorax; equal numbers from mated male and female flies) were analyzed.

\section{Exposure of flies to compounds, locomotion (climbing), and longevity assays}

The transgene (Tg) expression inducer RU486 (Sigma Aldrich) was directly added to the flies' culture medium. Doses and duration of flies' exposure to compounds are indicated in figure legends. The locomotion (climbing) and longevity assays were done as described previously [27].

\section{Genomic DNA extraction and conventional PCR analyses}

To verify the establishment of transgenic flies carrying more than one $\mathrm{Tg}$, genomic DNA from transgenic larvae or flies' tissues was extracted with the Genomic DNA Kit of Thermo Scientific (\#K0512) and conventional PCR was performed. Primers were designed using the primer-BLAST tool (http:// www.ncbi.nlm.nih.gov/tools/primer-blast/) and were the following:

Valium20-F: ACCAGCAACCAAGTAAATCAAC, Valium20-R: TAATCGTGTGT GATGCCTACC; cncC/Nrf2-F: TGGAATTGGGCACCCATGGCG, cncC/Nrf2-R: AGT TTGAGTACGTCGTTCAACA.

\section{RNA extraction, CDNA synthesis, and quantitative Real Time} PCR (Q-PCR) analyses

Total RNA extraction from Drosophila tissues, cDNA preparation and Realtime Q-PCR were performed as described before [27]. Used primers have been reported before [27]; for the Pink1/park genes the primers were as follows:

Pink1-F: ACAGCTGGTCTACAACATCC, Pink1-R: ACTGTAGGATCTCCGGACTG; park-F: TTCTGCCGCAATTGTCTGCAGG, park-R: GCATGCAACCGCCATCTCGCTC; The $R p L 32 / r p 49$ gene expression was used as a normalizer.

\section{Preparation of tissue protein extracts, immunoblot analyses} and detection of protein carbonyl groups

Adult or larvae tissues protein extracts preparation and immunoblotting analyses were performed as described previously [22]. For the detection of protein carbonyl groups, the OxyBlot protein oxidation detection kit (Millipore, s7150) was used.

\section{Measurement of ROS, proteasome, and cathepsins enzymatic activities in tissue extracts}

For ROS measurement dissected flies' tissues were incubated with fluorescent dye $\mathrm{CM}-\mathrm{H}_{2}$ DCFDA for $30 \mathrm{~min}$ at $25^{\circ} \mathrm{C}$ in the dark; the assay was performed as per manufacturer's instructions.

To measure proteasome activities, heads or somatic tissues were lysed on ice, with buffers suitable for $26 \mathrm{~S}$ proteasome isolation $(0.2 \%$ Nonidet $\mathrm{P}$ 40, $5 \mathrm{mM}$ ATP, $10 \%$ glycerol, $20 \mathrm{mM} \mathrm{KCl}, 1 \mathrm{mM}$ EDTA, $1 \mathrm{mM}$ DDT, and $20 \mathrm{mM}$ Tris, $\mathrm{pH}$ 7.6). Following centrifugation at $19,000 \times \mathrm{g}\left(4^{\circ} \mathrm{C}\right)$, supernatants ( $20 \mu \mathrm{g}$ of protein) were used to measure (after $30 \mathrm{~min}$ at $37^{\circ} \mathrm{C}$ ) the CT-L (LLVY) and C-L (LLE) enzymatic activities by recording the hydrolysis (excitation, $350 \mathrm{~nm}$; emission, $440 \mathrm{~nm}$ ) of the fluorogenic peptides $\mathrm{N}$ Succinyl-Leu-Leu-Val-Tyr-AMC (AMC = 7-amino-4-methylcoumarin) (BMLP802) and Z-Leu-Leu-Glu-AMC (BML-ZW9345) (Enzo Life Sciences; Farmingdale, NY, USA), respectively.

To measure cathepsins activity, heads or somatic flies' tissues were homogenized in extraction buffer ( $1 \mathrm{mM}$ dithiothreitol and $1 \mathrm{M}$ Tris, $\mathrm{pH} 4.0$ ) and the lysates were cleared at $19,000 \times g$ for $15 \mathrm{~min}\left(4^{\circ} \mathrm{C}\right)$. Twenty micrograms of protein were then incubated in the reaction buffer $(50 \mathrm{mM}$ sodium acetate, $8 \mathrm{mM}$ cysteine-hydrochloride, $1 \mathrm{mM}$ EDTA, $\mathrm{pH}$ 4.0) containing the substrate Z-Phe-Arg-AMC (BML-P139) (Enzo Life Sciences) for $30 \mathrm{~min}$ at $37^{\circ} \mathrm{C}$ and the fluorescence was recorded (excitation, $350 \mathrm{~nm}$; emission, $440 \mathrm{~nm}$ ). Assays were performed in adult flies; equal numbers of male and female flies were used.

\section{Tissue preparations for immunohistochemistry and confocal laser scanning microscope (CLSM) imaging}

Tissues were collected and dissected in PBS, fixed in $4 \%$ formaldehyde for $15 \mathrm{~min}$, washed in PBS containing $0.3 \%$ Triton X-100 and incubated with primary antibody overnight at $4{ }^{\circ} \mathrm{C}$. Secondary antibodies, DAPI (for nuclear visualization) or Phalloidin (for actin visualization) staining were applied for $1 \mathrm{~h}$ at RT and samples were mounted for viewing. Following dissection tissues were also stained with $100 \mu \mathrm{M}$ LysoTracker Red (as per manufacturer's instructions). The protocol used for the detection, imaging and processing of oxidized mitochondria was as described before [22]. Samples were viewed in a Digital Eclipse Nikon C1 (Nikon, Melville, NY, USA) CLSM equipped with $40 \times 1.0 \mathrm{NA}$ differential interference contrast (DIC), and $60 \times 1.4$ NA DIC Plan Apochromat objectives; image capturing was done using the EZC1 acquisition and images were analyzed with the CLSM software (Nikon Inc.). Z-stacks with a step size of $0.4 \mu \mathrm{m}$ were taken using identical settings. Each stack consisted of $28-30$ plane images; $\sim 10$ animals per genotype were analyzed and representative images are shown. ImageJ JACOP plugin was used for estimating co-localization of lysosomes (Lamp1-GFP) and acidic vesicles stained with LysoTracker Red.

\section{Antibodies and probes}

Primary antibodies against the Drosophila proteasome subunits 20S-a (sc65755), p42A/Rpn7 (sc-65750) and p54/Rpn11 (sc-65746); the antiUbiquitin (Ub) (sc-8017) and the HRP-conjugated secondary antibodies were purchased from Santa Cruz. Primary antibodies against the mitochondrial proteins ATP5a/blw (ab14748) and Ndusf3 (ab14711) were purchased from Abcam. Anti-Gapdh (G9545) and anti-Flag (F3165) antibodies were obtained from Sigma Aldrich. The anti-Rabbit-lgG Rhodamine (TRITC) conjugated antibody (711-025-152) and Alexa Fluor ${ }^{\circledR}$ 
488 AffiniPure Goat Anti-Rabbit IgG (111-545-003) were from Jackson ImmunoResearch. DAPI (D1306), LysoTracker Red (L7528) and Phalloidin (P3457) were from Molecular Probes ${ }^{\mathrm{TM}}$-Thermo Fisher Scientific. AntiTyrosine Hydroxylase (anti-TH, AB152) was purchased from Merck Millipore and the Gabarap (the fly ortholog of Atg8a; hereafter referred to as Atg8a/ Gabarap) antibody (\#13733) was from Cell Signaling Technology, Inc.

\section{Mitochondria isolation and measurement of mitochondrial respiration}

Mitochondria were isolated as described before [22]. The protein content of isolated mitochondria was measured by the Bradford method and mitochondrial respiration was determined using a Clark-type oxygen electrode connected to a computer-operated Oxygraph control unit (Hansatech Instruments, Norfolk, UK) as described [22]. The respiratory control ratio (RCR) was calculated as the ratio of state 3 to state 4 (ST3:ST4).

\section{Statistical analyses}

Experiments were performed at least in triplicate (for each biological replicate, $n \geq 3$ ). Assays were done after pooling isolated male/female tissues from 10 to 20 flies, unless otherwise stated. For statistical analysis, the GraphPad Prism 7.0, the MS Excel and the Statistical Package for Social Sciences (IBM SPSS; version 23.0 for Windows, NY, USA) were used. Statistical significance was evaluated using Student's $t$-test. Data points correspond to the mean of the independent experiments and error bars denote standard deviation (SD); significance at $P<0.05$ or $P<0.01$ is indicated in graphs by one or two asterisks, respectively. Gene expression was plotted vs. the respective control set to 1 ; in all other cases (unless otherwise indicated) control values were set to 100 .

For flies' survival curves and statistical analyses, the Kaplan-Meier procedure and log-Rank (Mantel-Cox) test were used; significance was accepted at $P<0.05$. Statistical analyses for all presented longevity experiments are reported in Supplemental Table S1.

\section{RESULTS \\ KD of park or Pink1 genes in young flies mobilizes proteostatic responses, increases oxidative load, causes mitochondrial aggregation in flies' tissues, and accelerates age-related degenerative phenotypes}

Initially we studied the functional crosstalk of mitostatic and proteostatic modules in young flies' tissues, by modulating the expression of park or Pink1 genes. We thus ubiquitously $\left(\mathrm{Gal} 4^{\mathrm{Tub}}\right)$ overexpressed or knocked down park or Pink1 in Drosophila. To assess the Tgs overexpression (OE)-mediated molecular responses we initially studied the genomic responses in transgenic flies' somatic tissues. Gene expression analyses showed similar readouts mainly after park or Pink1 KD; these referred to induction of proteasomal genes, as well as to upregulation of $\mathrm{cncC} / \mathrm{Nrf2}$ and the mitophagy receptor ref(2)P (the fly ortholog of mammalian SQSTM1/p62; hereafter referred to as $\operatorname{ref}(2) P / p 62)$, but not of Atg8a (the LC3 ortholog in Drosophila) (Fig. 1A). Some notable exceptions to similar genomic responses were foxo and mitochondrial genes, which tended to be induced in park KD but were downregulated in Pink1 KD mutant flies (Fig. 1A).

We also found that park and Pink 1 are downregulated in middle aged (30-33 days old) and aged ( $>45$ days old) wild type flies (Fig. $1 B$ ), and thus their induced ubiquitous or tissue specific KD in young flies mimics an age-related condition. Since deregulation of park and/or Pink1 expression relates to human degenerative diseases [10], we focused our studies on park, Pink1 KD-mediated functional outputs.

In support to gene expression analyses, 20S- $a$ proteasomal proteins were upregulated after park or Pink1 KD (Fig. 1C); yet park $\mathrm{KD}$ led to the accumulation of ubiquitinated (Ub) and oxidized [carbonylated (DNP)] proteins (Fig. 1C and Supplemental Fig. 1a). Although similar, these effects were milder in Pink1 RNAi expressing mutant flies (Fig. 1C and Supplemental Fig. 1a). Studies in isolated mitochondria showed that neither park nor Pink1 KD affected overall mitochondrial ubiquitination (not shown).
Downstream to these effects, prolonged (25 days) ubiquitous $\left(\mathrm{Gal}^{\mathrm{Tub}^{\mathrm{Tu}}}\right.$ ) park KD upregulated proteasomal activities (Fig. 1D) and either park or Pink1 KD significantly increased oxidative load in transgenic flies' somatic tissues (Fig. 1E). Furthermore, by using the redox sensitive GFP (roGFPs) probe to study the in vivo oxidative load in the mitochondria of park and Pink1 KD flies, we found that both transgenic fly lines tended to have increased oxidation load (Supplemental Fig. 1b, c). Also, Pink1 KD enhanced cathepsins activity (Fig. 1F), upregulated lysosomes number (Fig. 1G), and induced Atg8a/Gabarap protein and its lipidated form expression levels (Fig. 1H); the ALP-related effects were marginal in park KD mutant flies (Fig. 1F, G).

Analysis of mitochondria structure after muscle targeted $\left(G\right.$ al $\left.4^{\text {Mef2 }}\right) \mathrm{Tg}$ expression using the Mito-GFP reporter revealed the disruption of the mitochondrial network, and the increased formation of mitochondrial aggregates after park or Pink1 KD (but not their OE; not shown) in both larvae (Fig. $2 \mathrm{~A}_{1}$ ) and adult flies (Fig. 2 $\mathrm{A}_{2}$ ) muscles; notably this genetic intervention did not significantly affect mitochondria length (Supplemental Fig. 1d). We also found the wing posture to be disrupted in young flies after muscle-targeted (not shown) or ubiquitous (Gal4 ${ }^{\text {Tub }}$ ) (Fig. 2B) park or Pink1 KD. This degenerative effect coincided with a significant reduction in locomotion (climbing) activity of the transgenic young flies (Fig. 2C), disruption in the organization of muscle intestine fibers (Fig. 2D) and, especially in the park KD mutant flies, accelerated aging (Fig. 2E and Supplemental Table S1).

Thus, ubiquitous park or Pink1 KD in young flies impacts on mitostatic, proteostatic and antioxidant machineries causing their extensive readjustment, while sustained park or Pink1 KD results in accumulating mitochondrial aggregates, neuromuscular degenerative phenotypes, and accelerated aging.

Neuronal-specific or muscle-specific park, Pink1 KD disrupts proteostasis and suppresses mitophagy

To assess the effects of park or Pink1 genes expression silencing specifically in the brain, we expressed the park or Pink1 RNAi Tgs in flies' neuronal tissues (Gal4 ${ }^{\text {Elav }}$ ). The dopaminergic (DA) network in the fly is composed of well-characterized neuron clusters, i.e., the PPM1, PPM2, PPM3, PPL1, PPL2, and VUM [28] and considering that DA neurodegeneration is a major sign of PD we immunoassayed the DA neuronal marker tyrosine hydroxylase (TH) in dissected brains after park or Pink1 KD. Following prolonged (25 days) expression of the Tgs in neuronal tissues, we found a significant decrease of the DA neurons number in the PPL1 cluster (Fig. 3A), which has consistently been reported to be affected in PD fly models [29]. Further, we observed collapsed proteostasis being marked by reduced proteasomal (Fig. 3B) and lysosomal (Fig. 3C) cathepsins activities; elevated ROS levels (Fig. 3D) and a significant decrease in the expression levels of proteostatic/ mitostatic genes (with the notable exception of the chaperone Hsp70 gene induction after park KD; Fig. 3E1) and of 20S-a proteasomal proteins (Fig. 3E2) in isolated brains of park or Pink1 $\mathrm{KD}$ transgenic flies.

To investigate the contribution of park and Pink1 proteins in neuronal mitophagy turnover rates we used the Mito-QC reporter; a tandem GFP-mCherry fusion protein of the outer mitochondrial membrane, which turns red due to selective quenching of GFP in the acidic microenvironment of the lysosome. The spectral shift results in "red-only" puncta which mark mitolysosomes [27, 30]; in these assays quantification of mitolysosomes was performed using whole brain regions after CLSM Z-stack acquisition. Mitophagy rates in control larvae were developmentally regulated (not shown) and mitophagy was more evident in live vs. fixed neuronal tissues (Supplemental Fig. 2). Our studies with the Mito-QC reporter in control flies showed that nervous tissues express high basal rates of mitophagy (Fig. 3F) and that park KD suppressed mitophagy, as was evident by the significant reduction (vs. controls) of 

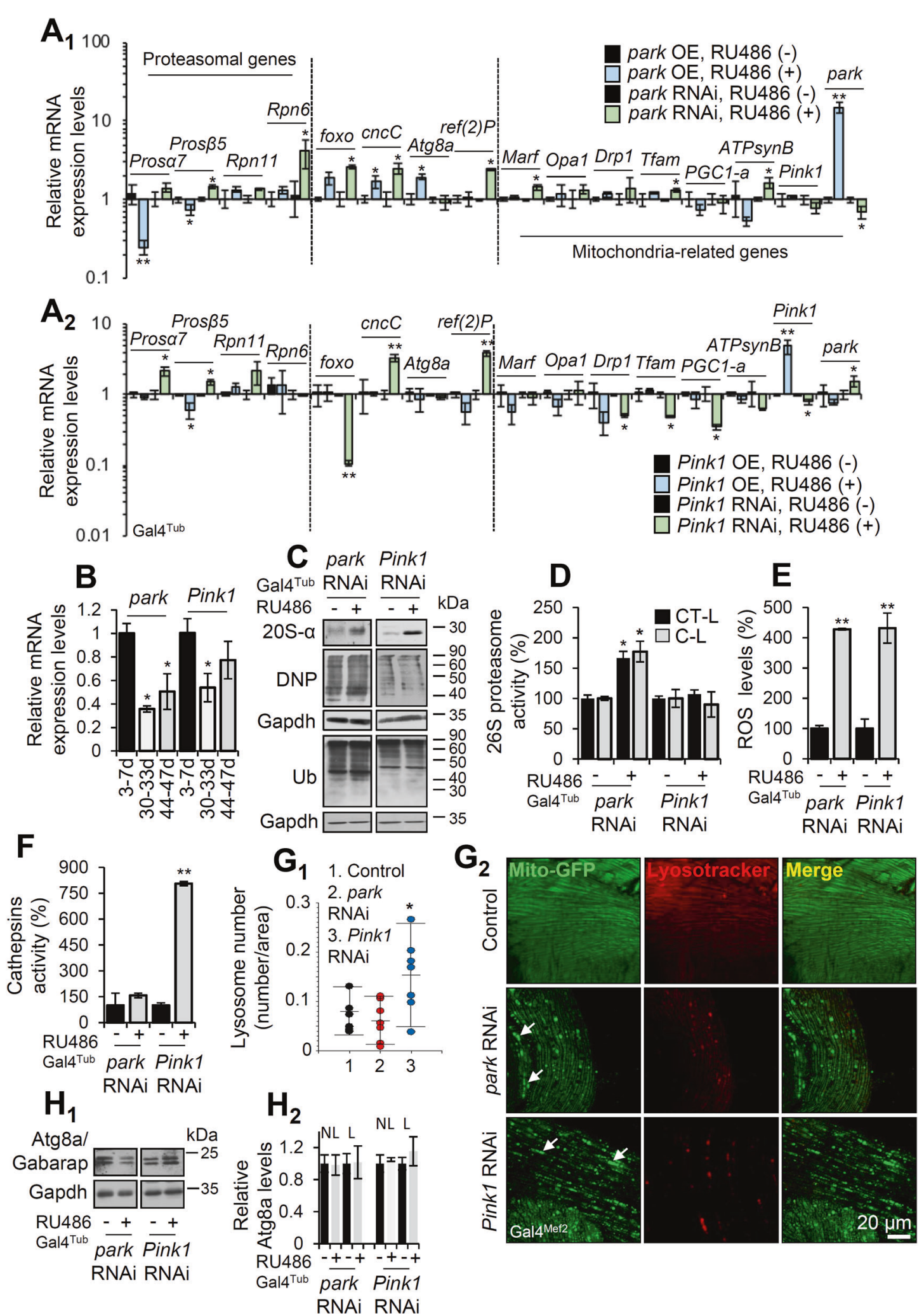

mitolysosomes in both larvae and adult brains (Fig. 3F, G). Pink1 $\mathrm{KD}$ in adult flies (but not in larvae) tended to also reduce mitophagy; yet the effect did not reach statistical significance (Fig. 3F, G). Consistently, neuronal specific KD of park, and to a lesser extent of Pink1, accelerated aging of transgenic flies (Supplemental Fig. 3 and Supplemental Table S1). Similarly to

nervous tissues, mitophagy rates in muscles were larvae stagedependent and more evident in live vs. fixed tissues in control flies (Supplemental Fig. 2), while as in the brain, muscletargeted (Gal4 ${ }^{\text {Mef2 }}$ ) park, or Pink1 KD decreased mitolysosomes number in larvae muscles, with park KD displaying again the stronger phenotype (Supplemental Fig. 4). 
Fig. 1 Induced KD of the mitophagy-related park or Pink1 genes in flies' tissues mobilizes proteostatic responses. A Relative mRNA expression of proteasomal subunits, transcription factors (foxo, cncC/Nrf2), autophagy related (Atg8a, ref(2)P/p62) and mitochondrial genes after inducible OE or KD of $\left(\mathbf{A}_{1}\right)$ park or $\left(\mathbf{A}_{\mathbf{2}}\right)$ Pink1 genes. B Relative mRNA expression levels of park and Pink1 genes in middle aged (30-34 days old) and aged (44-47 days old) wild type flies vs. young (3-7 days old) flies. C Immunoblot analyses ( $n=2)$ after probing the shown transgenic flies' tissues protein samples with antibodies against $20 \mathrm{~S}-\alpha$ proteasomal subunits, total ubiquitin (Ub) and protein carbonylation (dinitrophenol, DNP). D, E Relative (\%) $26 \mathrm{~S}$ chymotrypsin-like (CT-L) and caspase-like (C-L) like proteasome activities (D) and ROS levels (E) of the shown transgenic lines. F Relative (\%) cathepsins activity. G Lysosome quantification $\left(\mathbf{G}_{1}\right)$ and CLSM co-visualization of mitochondria (Mito-GFP) and lysosomes (LysoTracker Red) $\left(\mathbf{G}_{2}\right)$ in adult flies' muscle tissues after muscle-targeted (Gal4 ${ }^{\text {Mef2 }}$ ) KD of park or Pink1 genes. $\mathbf{H}$ Immunoblot analyses of $\left(\mathbf{H}_{\mathbf{1}}\right)$ somatic tissues probed with anti-Atg8a/Gabarap $(n=2)$ and $\left(\mathbf{H}_{\mathbf{2}}\right)$ relative protein quantification $(\mathrm{NL}$ non lipidated, L lipidated Atg8a/Gabarap). Flies were exposed to $320 \mu \mathrm{M}$ RU486 in (A) for 7 days and in (C-F) for 25 days. Gene expression in (A, B) was plotted vs. control set to 1 (RpL32/rp49 gene was used as reference). Gapdh probing in $\left(\mathbf{C}_{\mathbf{1}} \mathbf{H}_{\mathbf{1}}\right)$ was used to demonstrate equal protein loading. Bars, $\pm \mathrm{SD} ; n=3,{ }^{*} P<0.05 ;{ }^{* *} P<0.01$.

Taken together these findings highlight the toxic effects induced in young flies' neuronal or muscle tissues (likely due to disrupted proteostasis and unbalanced mitophagy) following park or Pink1 KD.

\section{OE of cncC/Nrf2 normalizes [independently to ref(2)P/p62)] proteostatic and mitostatic modules functionality in park or Pink1 KD transgenic flies' tissues}

Given our recent findings showing that $\mathrm{cncC} / \mathrm{Nrf2}$ is a transcriptional regulator of mitochondrial genes [21], we then examined if park and/or Pink1 genes are also regulated by $\mathrm{cncC} / \mathrm{Nrf2}$. We found that $c n c C / N r f 2$ OE induced park and Pink1 (along with ref(2)P/p62) expression levels, which were suppressed after cncC/Nrf2 KD (Supplemental Fig. 5). Thus, cncC/Nrf2 is not only a positive regulator of $\operatorname{ref}(2) P / p 62$, park, and Pink1 genes expression, but it also maintains their basal expression levels.

Considering that Nrf2 function is impaired in mitochondriarelated disorders, including PD [20], and the high oxidative load in park, Pink1 KD flies, we OE cncC/Nrf2 ubiquitously (Supplemental Fig. 6a) (Gal4 ${ }^{\text {Tub }}$ ) in park and Pink1 RNAi expressing flies. Initially, we found that the endogenous $\mathrm{cncC} / \mathrm{Nrf2}$ in Drosophila, is expressed at all developmental stages and in most Drosophila tissues (Supplemental Fig. 6b). OE of $\mathrm{cncC} / \mathrm{Nrf2}$ in park or Pink1 KD tissues upregulated proteasomal subunits (Prosa7, Pros $\beta 5$, and Rpn11), the mitophagy receptor $\operatorname{ref}(2) P / p 62$ and Atg8a genes (Fig. 4A). Consistently, it also augmented 20S-a proteasomal proteins expression (Fig. 4B) and proteasomal activities (Fig. 4C); it also reduced ROS levels (Fig. 4D) and mitochondria oxidative load (Supplemental Fig. 7) as compared to transgenic flies solely expressing park and Pink1 RNAi. Remarkably, OE of cncC/Nrf2 in the park or Pink1 KD background also upregulated the expression of mitochondrial genes [i.e., Marf, Drp1, and PGC1- $a$ (the fly ortholog of mammalian PPARGC1A; peroxisome proliferatoractivated receptor gamma coactivator 1-alpha)] (Fig. 4E). In addition, cncC/Nrf2 OE upregulated the expression levels of mitochondrial complex V protein, ATP5a/blw [but not of complex I (Ndufs3)] (Supplemental Fig. 8a), and it also rescued mitochondrial respiration defects (Fig. 4F) and flies wing posture (Supplemental Fig. 8b).

We thus investigated whether $\mathrm{cncC} / \mathrm{Nrf2}$ OE could revert tissue specific disrupted mitostasis in park or Pink1 KD flies. We found that muscle-targeted (Gal4 ${ }^{\text {Mef2 }}$ ) cncC/Nrf2 OE eliminated Mito-GFP aggregates (Fig. 5A, B) and increased lysosomes number (Lysotracker Red staining; Fig. 5C, D) in both park and Pink1 KD larvae. In support, ubiquitous $\mathrm{CncC} / \mathrm{Nrf2}$ overexpressing flies in the park or Pink1 KD background had increased cathepsins activities (Fig. 5E and Supplemental Fig. 8c), suggesting ALP stimulation. Moreover, cncC/Nrf2 OE increased co-localization of Atg8a/ Gabarap with mitochondria in larvae (not shown) and adult flies' muscles (Gal4 ${ }^{\text {Mef2 }}$ ) (Fig. 5F) suggesting mitophagy stimulation. Consistently, muscle-specific expression of the Mito-QC reporter showed that $\mathrm{cncC} / \mathrm{Nrf2} \mathrm{OE}$ significantly enhanced (vs. controls) mitophagy in park and Pink1 KD flies (Fig. 5G).
Interestingly, ref(2)P/p62 KD in either Park RNAi, cncC/Nrf2 OE or Pink1 RNAi, cncC/Nrf2 OE transgenic flies did not revert the $\mathrm{cncC} /$ Nrf2 OE-mediated elimination of the Mito-GFP aggregates (Supplemental Fig. 9). Also, ref(2)P/p62 KD in solely park or Pink1 RNAi expressing flies tended to further increase the number of mitochondria aggregates in park (Supplemental Fig. 10), but not Pink1 (not shown), KD flies indicating that park is more ref(2)P/p62dependent for mitophagy induction. Therefore, in this biological setting $\mathrm{cncC} / \mathrm{Nrf2}$-induced mitophagy is likely independent of the autophagy/mitophagy adapter ubiquitin-binding protein ref(2)P/ p62.

Thus, cncC/Nrf2 OE activated proteostatic modules and reverted (independently to ref(2)P/p62) the formation of Mito-GFP aggregates in park or Pink1 RNAi expressing flies.

\section{cncC/Nrf2 activation alleviates the neuromuscular} degeneration phenotypes of park or Pink1 KD mutant flies Finally, we asked whether $c n c C / N r f 2$ OE could also rescue the neuromuscular degeneration phenotypes manifested in park or Pink1 KD flies. Gene expression analyses in isolated brains after neuronal-targeted (Gal4 ${ }^{\text {Elav }}$ ) co-expression of the Tgs showed that targeted $\mathrm{cncC} / \mathrm{Nrf2} \mathrm{OE}$ upregulated proteasomal subunits and mitochondrial genes but not $\operatorname{ref}(2) P / p 62$ and Atg8a (Fig. 6A; see also Fig. $3 \mathrm{E}_{1}$ ), indicating tissue-specific responses after $\mathrm{cncC} / \mathrm{Nrf2}$ OE. Importantly, PGC1-a was also induced by $\mathrm{cncC} / \mathrm{Nrf2} \mathrm{OE}$ (Fig. 6A). Previous reports have shown that apart from a master regulator of mitochondria biogenesis, $P G C 1-a$ also exerts a neuroprotective role [31]. Also, cncC/Nrf2 OE in nervous tissues increased proteasome activities (Fig. 6B), reduced oxidative load (Fig. 6C) and as found in muscles, enhanced the overall mitophagy turnover rates in park or Pink1 KD flies' brain (Fig. 6D). In support, it prevented loss of DA neurons in the brain of park or Pink1 KD flies (Fig. 6E), rescued the park, Pink1 KD mutant's locomotion defects (Fig. 6F) and tended to improve, especially in the park KD background which showed the more severe phenotype, young flies' health-span (Supplemental Fig. 11 and Supplemental Table S1). Yet, consistently to our recent findings showing that prolonged $c n c \mathrm{C} / \mathrm{Nrf2} \mathrm{OE}$ in the fly can be toxic [21] the overall longevity of these transgenic flies was not (vs. controls) improved (Supplemental Fig. 11 and Supplemental Table S1).

Taken together, these data support the notion that activation of the druggable cncC/Nrf2 pathway can improve the severe effects of age-related park or Pink1 reduced functionality.

\section{DISCUSSION}

UPP and ALP play a critical role in regulating cellular functionality and viability and the decline of their activity is a hallmark of aging, and of multiple age-related diseases including neuromuscular degenerative disorders [16, 32]. Seemingly, there is an extensive functional wiring between antioxidant, proteostatic, and mitostatic modules [21, 22, 29, 33] and both antioxidant and proteostatic pathways curate mitochondrial functionality by 


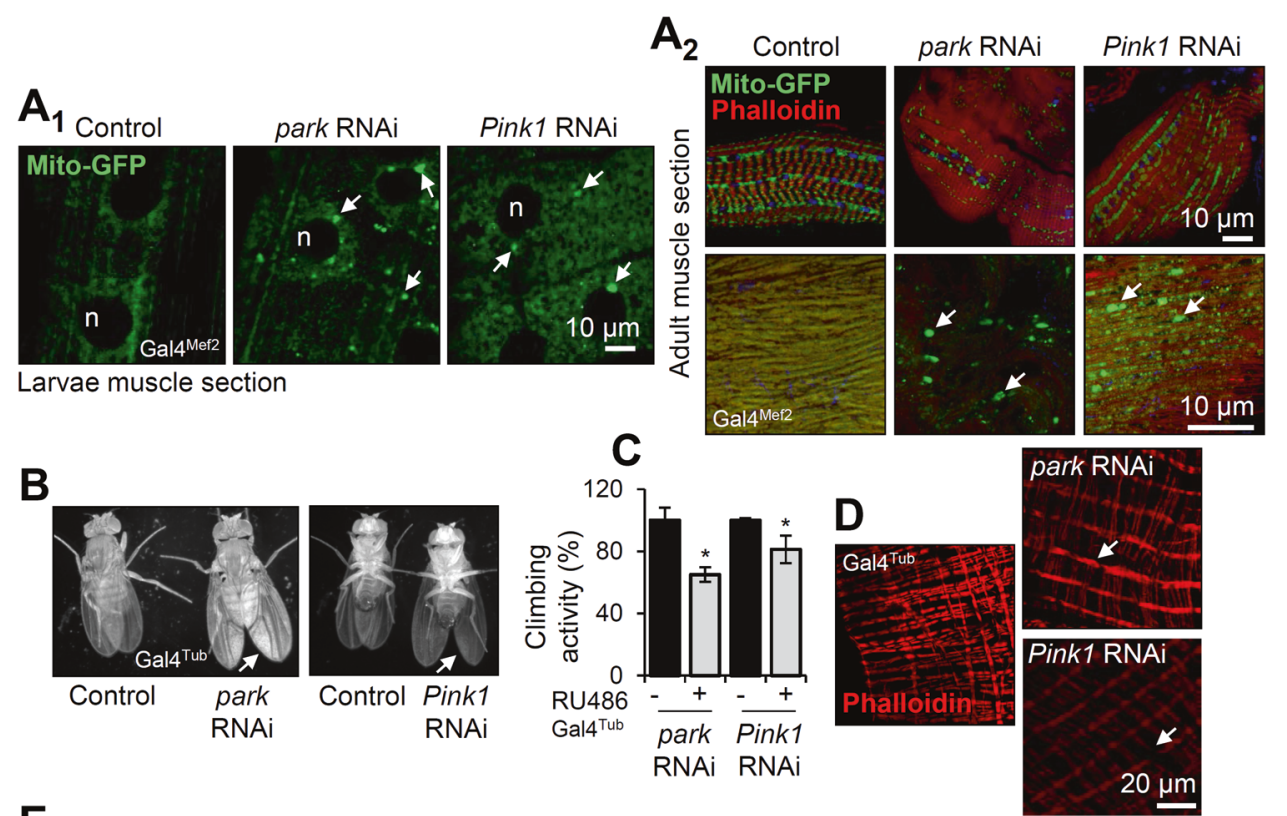

$\mathbf{E}$

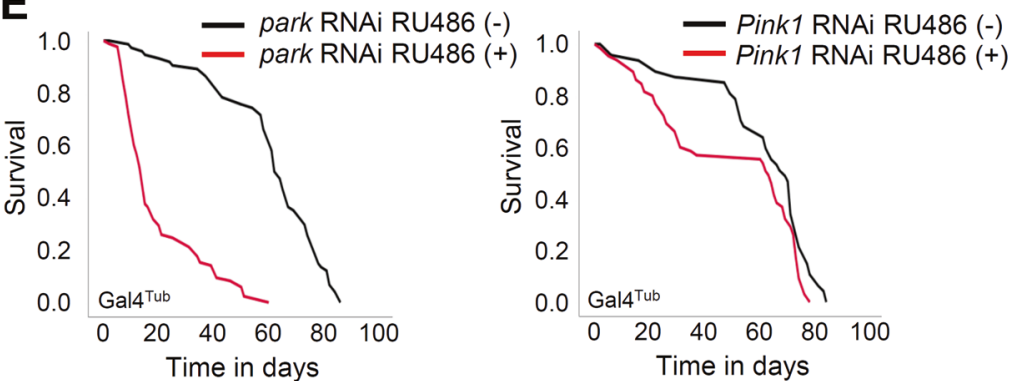

Fig. 2 Expression of park or Pink1 RNAi accelerates degenerative age-related phenotypes in transgenic flies. A CLSM visualization of muscle mitochondria after muscle targeted (Gal4 ${ }^{\text {Mef2 }}$ ) KD of park or Pink1 genes in larvae $\left(\mathbf{A}_{1}\right)$ and adult flies $\left(\mathbf{A}_{2}\right)$ muscles; Mito-GFP was used to visualize mitochondria, phalloidin (red) for muscle actin and DAPI (blue) for cell nuclei. B Light stereoscope imaging of wing posture in control [RU486 (-)], park and Pink1 RNAi expressing flies. C Relative locomotion (climbing) activity of 25 days old flies of the shown genotypes after ubiquitous (Gal4 ${ }^{\text {Tub }}$ ) Tg expression. D Intestine muscle staining with phalloidin (red) after park or Pink1 muscle targeted (Gal4 ${ }^{\text {Mef2 }}$ ) KD. E Longevity curves of the shown transgenic fly lines after ubiquitous (Gal4 ${ }^{\text {Tub }}$ ) park or Pink1 KD. Log-rank, Mantel-Cox test: control vs. park RNAi $P<0.0001$, control vs. Pink1 RNAi $P<0.01$. Statistics of the longevity curves are also reported in Supplemental Table S1. Flies were exposed to $320 \mu \mathrm{M}$ RU486 for 25 days, except otherwise indicated. Bars, $\pm \mathrm{SD} ; n=3,{ }^{*} P<0.05$.

triggering adaptive repair responses, mitochondrial biogenesis, and/or even removal of the damaged organelle by mitophagy; insufficiency of these responses can induce cell death [34].

We report that park or Pink1 KD in young flies modulated proteostatic modules in a tissue-dependent manner; ubiquitously increased cell oxidative load (shown also after loss-off PINK1 function in SH-SY5Y human neuroblastoma cells [35]), and suppressed mitophagy in neuronal and muscle tissues causing mitochondrial aggregation and neuromuscular degeneration. Pink1 exploits the canonical mitochondrial import pathway and under physiological conditions faces proteasome degradation [36], while the E3 ubiquitin ligase Parkin is one of the numerous UPP components recruited at the OMM upon mitochondrial damage $[7,37]$. The mitochondrial localization of these components is another indication of the linkage between proteostaticmitostatic modules; a notion that is further supported by the finding that $\sim 62 \%$ of the mitochondrial proteome might be ubiquitinated for UPP degradation [33]. Notably, park KD led to increased proteasomal enzymatic activities, while, Parkinmediated mitophagy is reduced when proteasome is inhibited and proteasomes accumulate on mitochondria during Parkininduced mitophagy [38, 39]. Thus, proteasomal activities are tightly regulated as a response to altered functionality of Parkin. On the other hand, it has been reported a deficit in proteasome activity in Pink1 loss-of-function cells and it was suggested that this readout may relate to reduced ATP production that Pink1 lossof-function cells displayed [40]. Considering that both park and Pink1 KD mutant flies showed similar mitochondrial oxidative phosphorylation capacity, we hypothesize that the differential effects on proteasome activities in park, Pink1 KD flies may also relate to the different ubiquitination profiles that was induced in flies' tissues after Pink1 or park KD. Indeed, UPP degradation rates depend on proteome ubiquitination and the balance between ubiquitination/de-ubiquitination of the substrates [41].

Distinctly to park KD, downregulation of Pink1 upregulated Atg8a/Gabarap expression and lysosomal cathepsins activity, indicating the activation of autophagic responses; in support PINK1 knockout in mouse embryonic fibroblasts resulted in autophagy upregulation [42]. Yet, in either park or Pink1 KD mutant flies' tissues there was minimal co-localization between ALP components and mitochondria aggregates, further supporting the notion that mitophagy is regulated distinctly from bulk autophagy [43]. In our study in the fly model, Pink1 KD showed less intense phenotypes as compared to park KD. We hypothesize that this may relate to the fact that park functions downstream of Pink1 [28] and thus, in Pink1 RNAi flies the remaining active protein could partially activate park. Given also the differential readouts in terms of proteostatic responses and levels of tissue 

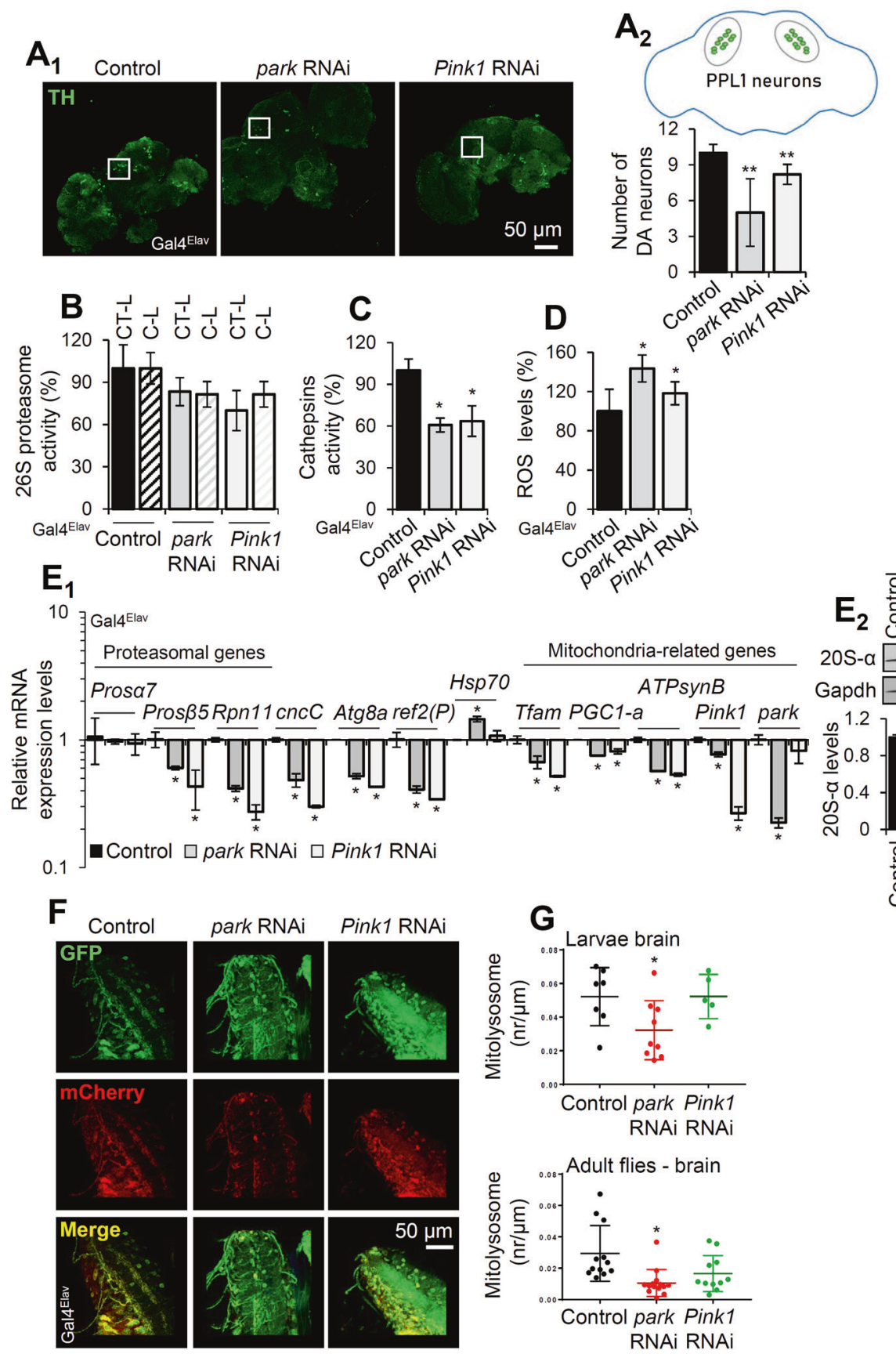

Fig. 3 Targeted KD of park or Pink1 in nervous tissues reduces brain DA neurons, downregulates proteostatic modules activities, induces oxidative stress and disrupts mitophagy. A CLSM viewing (projection, $Z$ stack) of whole-mount brains from middle aged flies showing ( $\mathbf{A}_{\mathbf{1}}$ ) DA neuron clusters marked with anti-TH immunostaining, and $\left(\mathbf{A}_{2}\right)$ quantification (vs. control) of DA PPL1 neurons in the brains of park or Pink1 KD transgenic flies. B-D Relative (\%) chymotrypsin-like (CT-L) and caspase-like (C-L) like proteasome activities (B), cathepsins activities (C), and ROS levels (D), in flies' brain after KD of park or Pink1 genes. $\mathbf{E}_{\mathbf{1}}$ Relative mRNA expression of proteasomal subunits, cncC/ Nrf2, autophagy-related (Atg8a, ref(2)P/p62), Hsp70 and mitochondria-related genes in transgenic flies of the indicated genotypes. $\mathbf{E}_{\mathbf{2}}$ Immunoblot analyses and relative quantification of the shown transgenic flies' brain samples probed with antibodies against $20 \mathrm{~S}-\alpha$ proteasomal subunits $(n=2)$. F CLSM visualization of the Mito-QC reporter GFP and mCherry signal in larvae nervous tissues, and (G) quantification of mitolysosomes after park or Pink1 KD in larvae (upper panel) and adult flies (lower panel) brain. A-G Tgs were expressed in nervous tissues (Gal4 ${ }^{\text {Elav }}$ ). Gene expression in $\left(\mathbf{E}_{1}\right)$ was plotted vs. control set to $1 ; R p L 32 / r p 49$ gene was used as reference. Gapdh probing in $\left(\mathbf{E}_{\mathbf{2}}\right)$ was used to demonstrate equal protein loading. Bars, $\pm S D ; n=3,{ }^{*} P<0.05 ;{ }^{*} P<0.01$.

oxidative load upon ubiquitous or tissue-targeted park or Pink1 $\mathrm{KD}$, it is likely that mitophagy displays (at least in flies) tissuedependent regulation. However, we cannot exclude that the noted park or Pink1 genes silencing effects could be partially independent of their role in mitophagy $[44,45]$.
Among the most challenging questions regarding mitochondrial removal is the role of Parkin/Pink1 in basal mitophagy. Similarly, to previous reports in C. elegans [46] we found high basal mitophagy rates in flies' tissues, which was nonetheless marked by tissue-dependency as basal mitophagy was higher in neuronal vs. 

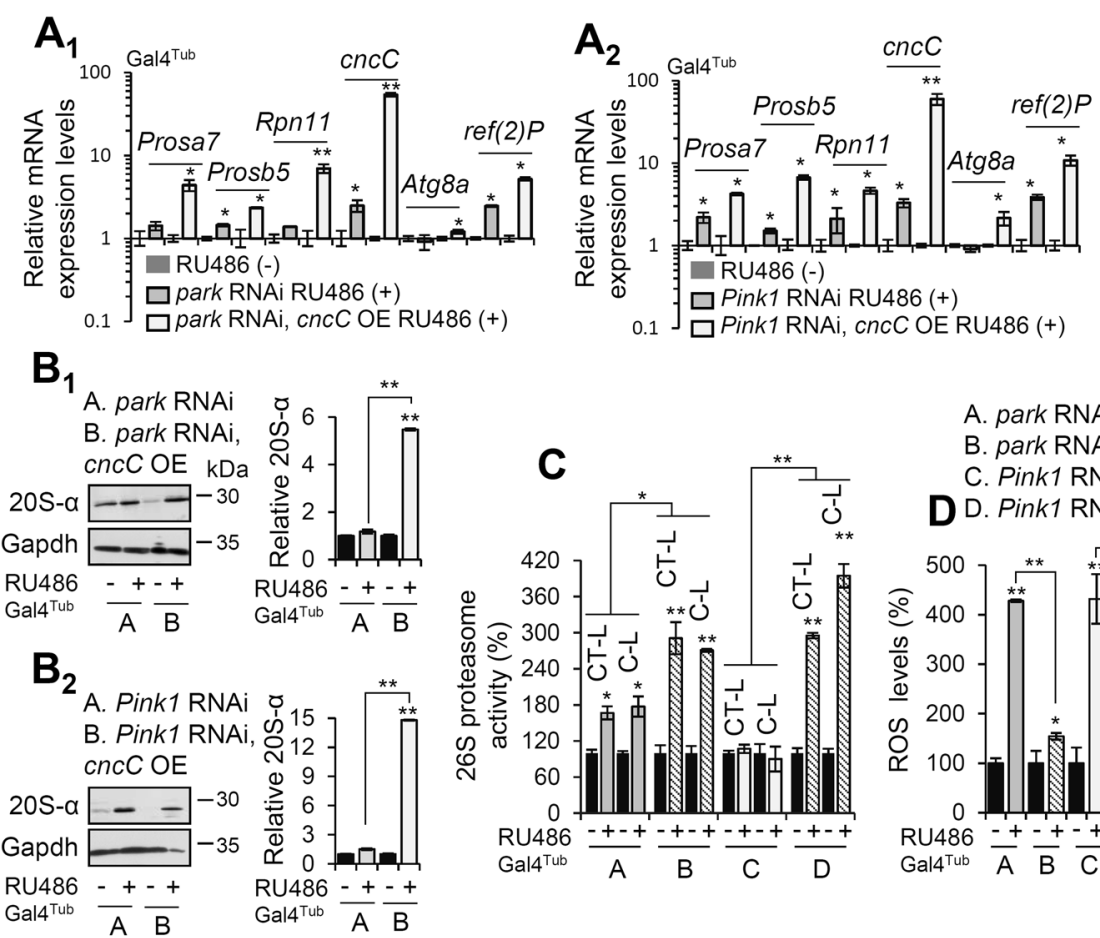
A. park RNAi
B. park RNAi, cncC OE
C. Pink1 RNAi

D D. Pink1 RNAi, cncC OE
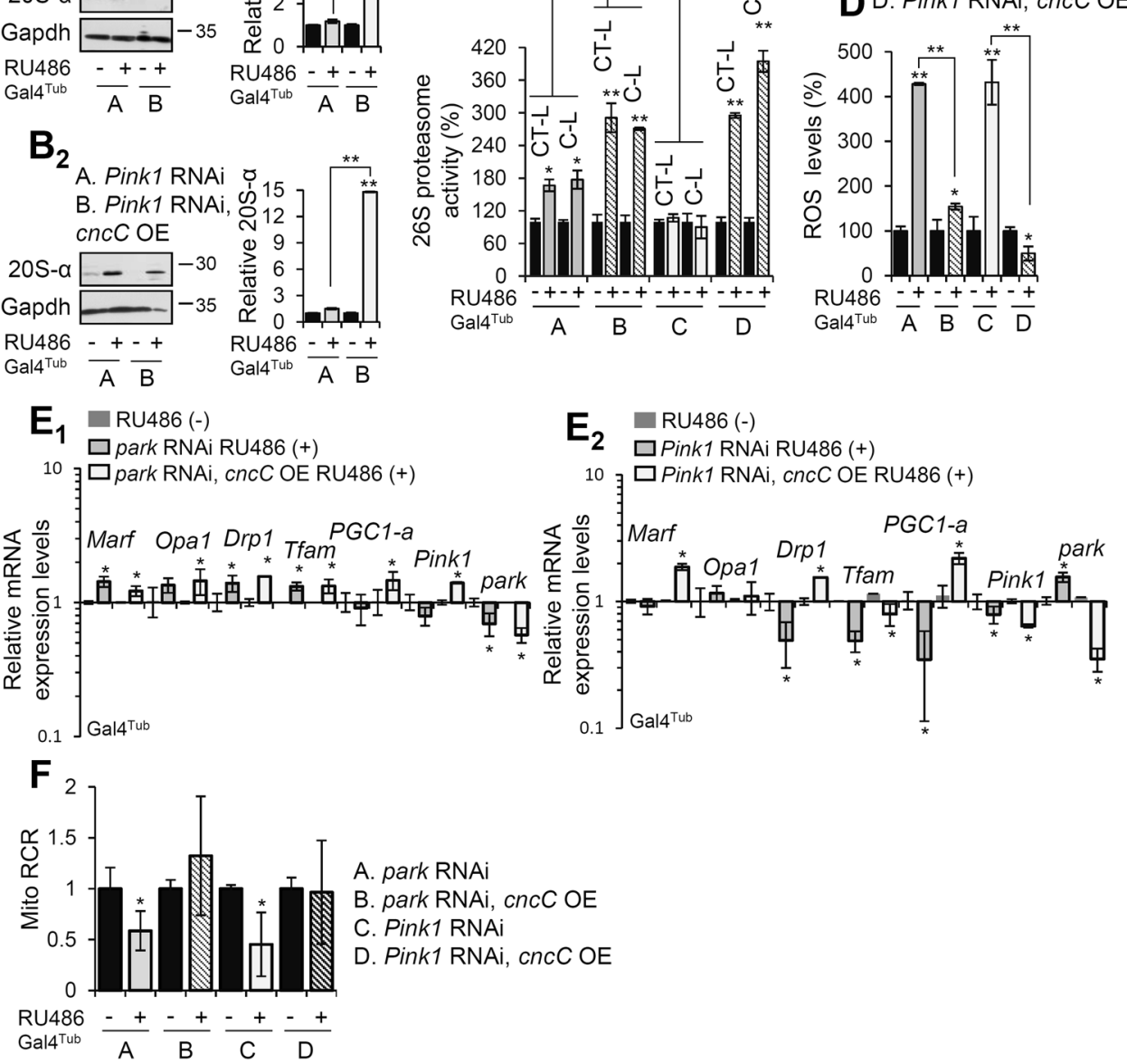

A. park RNAi

B. park RNAi, cncC OE

C. Pink1 RNAi

D. Pink1 RNAi, cncC OE

Fig. 4 Concomitant to ubiquitous park or Pink1 KD, CncC/Nrf2 OE upregulates UPP, suppresses oxidative stress and restores normal mitochondria respiration rates in transgenic flies' tissues. A Relative mRNA expression levels of proteasomal subunits (Prosa7, Pros $\beta 5$, Rpn 11$)$, cncC/Nrf2 and autophagy related (Atg8a, ref(2)P/p62) genes after $\mathrm{cncC} / \mathrm{Nrf2}$ OE at the park $\left(\mathbf{A}_{\mathbf{1}}\right)$ or Pink1 ( $\left.\mathbf{A}_{\mathbf{2}}\right)$ KD genetic background. B Immunoblot analyses and relative quantification of indicated transgenic flies' somatic tissues probed with antibodies against $20 \mathrm{~S}-\alpha$ proteasomal subunits. C, D Relative (\%) 26S chymotrypsin-like (CT-L) and caspase-like (C-L) proteasome activities (C), and ROS levels (D), following $\mathrm{cncC} / \mathrm{Nrf2} \mathrm{OE}$ in park or Pink1 KD flies. E Relative mRNA expression of mitochondrial genes in shown transgenic flies. F Quantitative analysis of isolated mitochondria respiratory capacity at the indicated transgenic fly lines. A-F Tgs were induced ubiquitously (Gal4 $4^{\text {Tub }}$ ); flies were exposed to $320 \mu \mathrm{M}$ RU486. Gene expression in (A, E) was plotted vs. control set to 1 (RpL32/rp49 gene was used as reference). Gapdh probing in (B) was used to demonstrate equal protein loading. Bars, $\pm S D ; n=3,{ }^{*} P<0.05 ;{ }^{*} P<0.01$.

muscle tissues. Previous studies suggested lack of basal mitophagy in Drosophila adults or pupae muscles [30]; yet we found increased rates of basal mitophagy in larvae muscles. This discrepancy likely relates to our observation that detection of mitophagy is affected by larvae developmental stage or tissue preparation. In support, lysosomes are abundant in flies' muscles [47] and it was suggested that their detection using probes should be made in live tissues [48]. Given the impact of tissue preparation or the developmental stage on mitophagy recording, the molecular mechanisms of this pathway, under basal or stress conditions, should be preferentially studied at in vivo models [46]. To this end, the analysis of transgenic animals expressing mitophagy reporters, such as Mito-QC, will further increase our understanding on mitophagy regulation.

Muscles were particularly sensitive to reduced park or Pink1 expression as we found that KD of Pink1 and especially of park reduced mitophagy events in muscles, a tissue of high metabolic demand. This phenotype explains the excessive accumulation of mitochondrial aggregates in transgenic flies' muscles along with the muscle related degenerative phenotypes seen in young flies, 

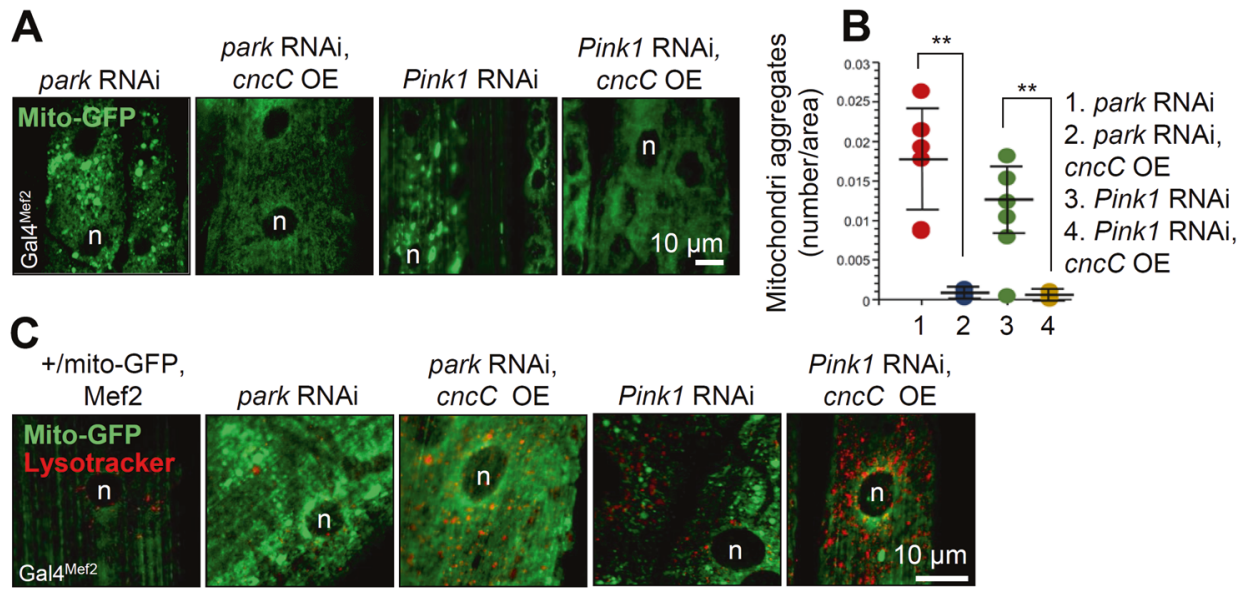

Pink1 RNAi, CncC OE
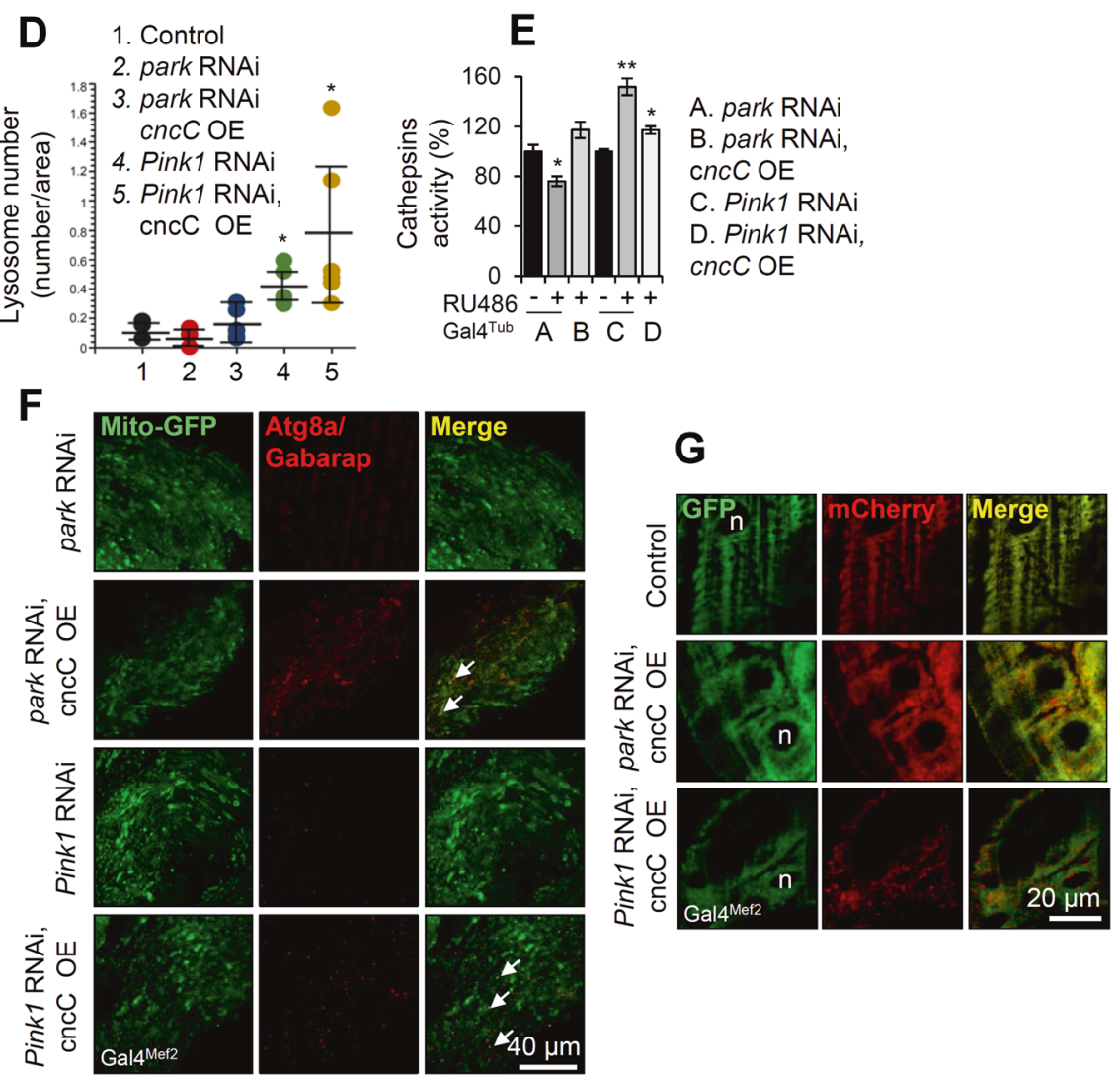

Fig. 5 cncC/Nrf2 OE enhances autophagy/mitophagy turnover rates in park or Pink1 KD flies. A CLSM viewing of mitochondria (Mito-GFP), and (B) quantification of mitochondria aggregates after muscle-targeted (Gal4 ${ }^{\text {Mef2 }}$ ) expression of the shown Tgs. C Mitochondria (Mito-GFP), lysosomes (LysoTracker Red) co-staining and (D) quantification of lysosomes in the shown flies' genotypes after muscle-targeted (Gal4 ${ }^{\text {Mef2 }}$ ) Tg expression. E Relative (\%) cathepsin activity in transgenic flies after ubiquitous (Gal4 ${ }^{\text {Tub }}$ ) park or Pink1 KD and concomitant cncC/Nrf2 OE. F CLSM viewing of mitochondria (Mito-GFP) and Atg8a/Gabarap immunostaining at the muscles (Gal4 ${ }^{\text {Mef2 }}$ driver) of shown adult transgenic flies. G CLSM visualization of the Mito-QC reporter GFP and mCherry signal at the indicated transgenic larvae wall muscle (Gal4 ${ }^{\text {Mef2 }}$ driver). Bars, \pm SD; $n=3,{ }^{*} P<0.05 ;{ }^{* *} P<0.01$.

i.e. disrupted wing posture and locomotion activity. Interestingly, our findings indicate that the role of Pink1 in Drosophila brain is dispensable for basal mitophagy, differently from park which (as in muscles) seemingly plays an important role on driving mitophagy events also in neuronal tissues. In support, the role of Pink1 in basal mitophagy is dispensable in several mouse tissues [49]. Up to date, little is known about the regulation of mitophagy in neurons and the functional involvement of the park/Pink1 pathway in this tissue. Given the high dependence of neurons on proper mitochondria functionality, one would assume that these cells have evolved additional pathways to remove damaged mitochondria [50]. Indeed, several studies have demonstrated the existence of park/Pink1-independent mitophagy cascades revealing multiple mitochondrial proteins (FUNDC1, BNIP3, NIX, and PHB2) or even lipids (e.g., cardiolipin) that mediate mitophagy in response to different stimuli [51-53].

Given the impact of mitochondrial dysfunction on neurodegeneration disorders we sought to rescue the Pink1 or park KDmediated deleterious effects. We found that concomitant to park or Pink1 KD, activation of the $\mathrm{cncC} / \mathrm{Nrf2}$ transcription factor $[20,21]$, ubiquitously induced the proteostasis network, suppressed oxidative stress and restored mitochondrial function and 


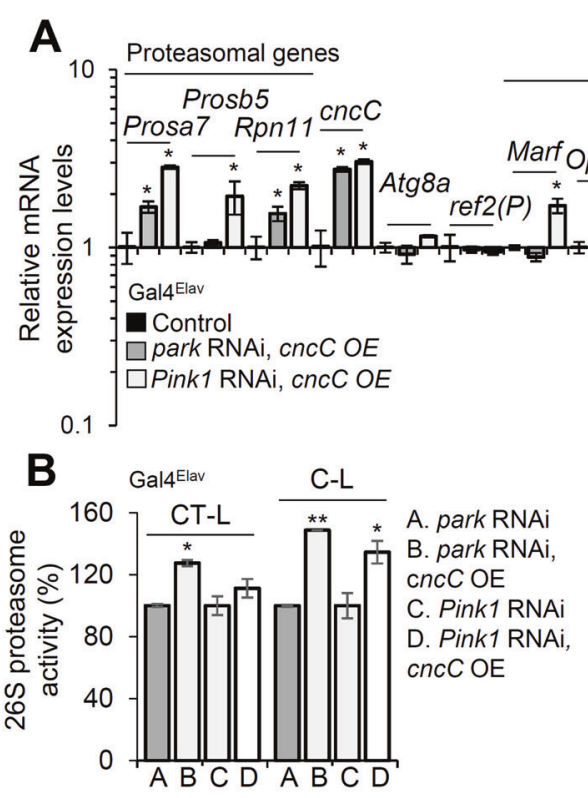

Mitochondria-related genes
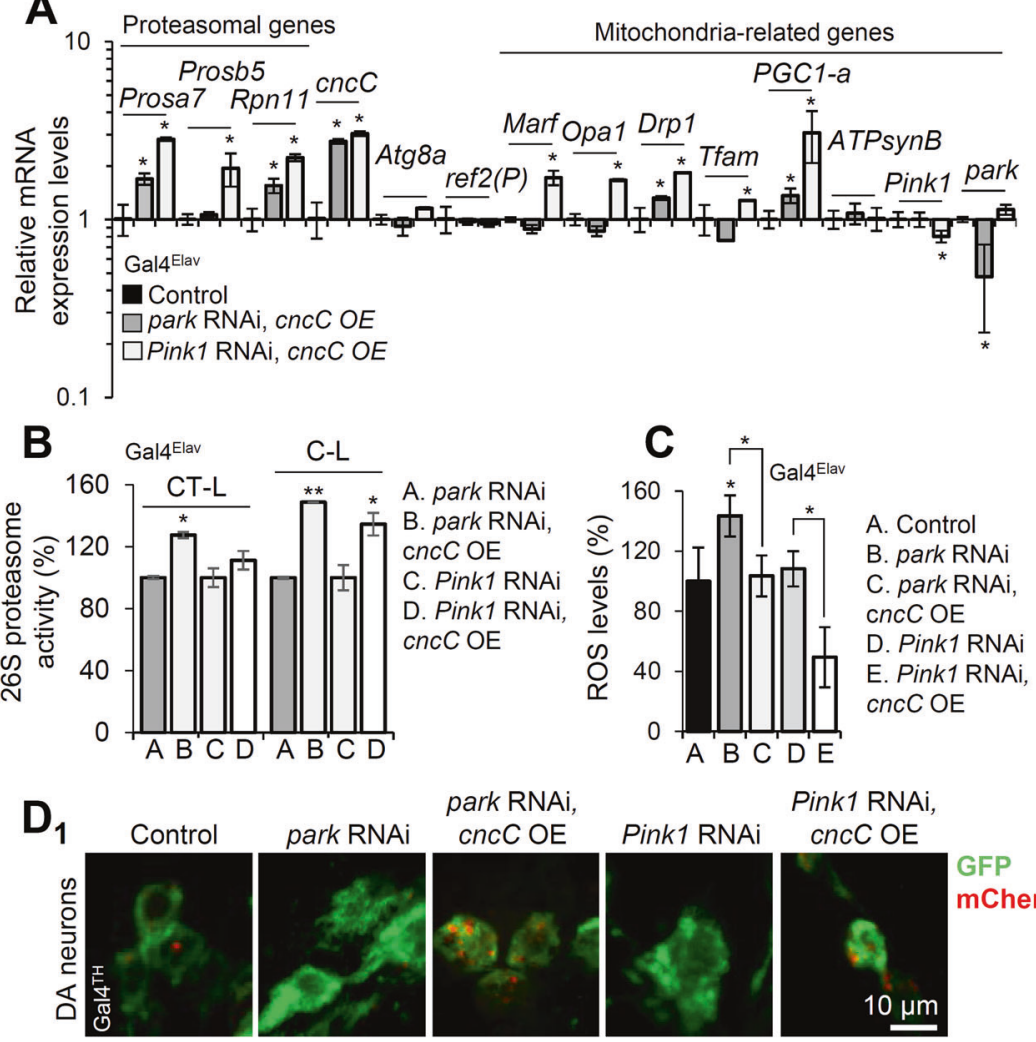

park RNAi,
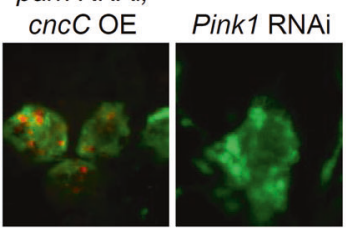

Pink1 RNAi,
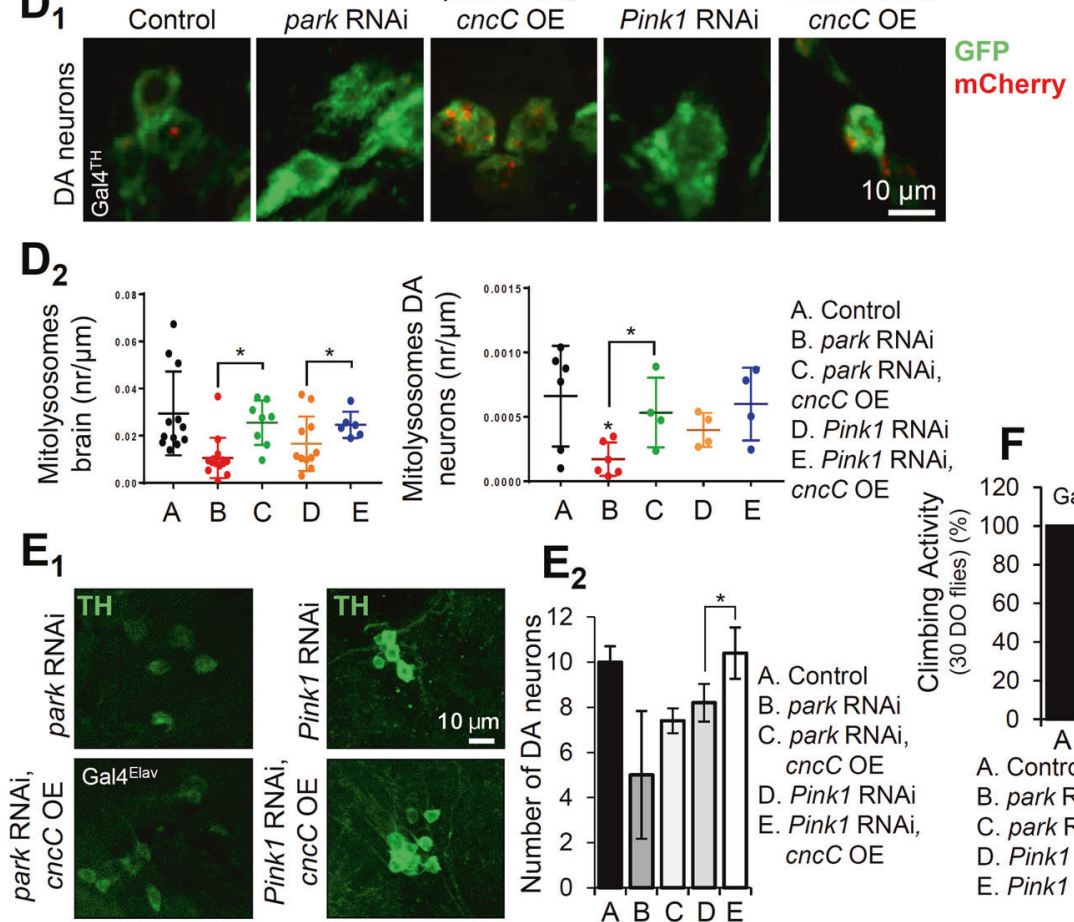

$E_{2}$
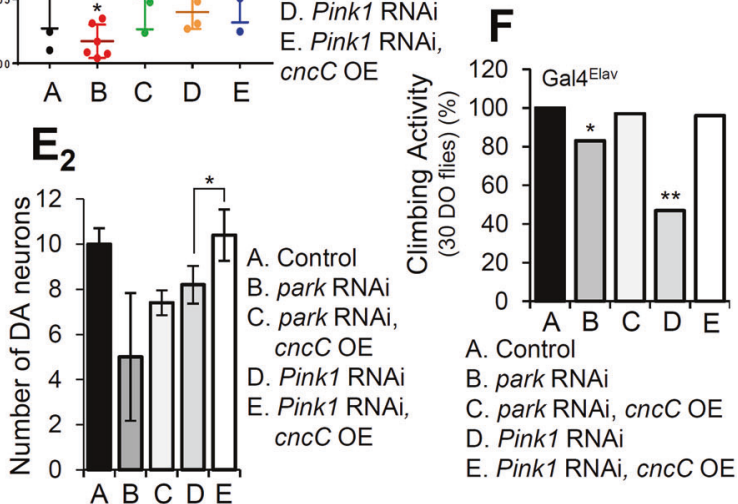

Fig. 6 OE of $\mathrm{cncC} / \mathrm{Nrf2}$ alleviates the neuromuscular degeneration phenotypes induced by nervous tissues-targeted park or Pink1 KD. A Relative mRNA expression of proteasomal subunits, cncC/Nrf2, autophagy related (Atg8a, ref(2)P/p62) and mitochondrial genes in dissected brains of the indicated transgenic flies. B, C Relative (\%) $26 \mathrm{~S}$ chymotrypsin-like (CT-L) and caspase-like (C-L) proteasome activities (B), and ROS levels (C), (head samples) after cncC/Nrf2 OE in Pink1 or park KD flies. $\mathbf{D}_{1}$ CLSM visualization of the Mito-QC reporter GFP-mCherry signal in brain DA neurons $\left(\mathrm{Gal}^{\mathrm{TH}}\right)$, and $\left(\mathbf{D}_{2}\right)$ quantification of brain $\left(\mathrm{Gal} 4^{\mathrm{Elav}}\right), \mathrm{DA}\left(\mathrm{Gal}^{\mathrm{TH}}\right)$ mitolysosome number of the shown adult transgenic flies. $\mathrm{E}_{1}$ CLSM images of DA PPL1 neurons (middle-aged flies) following staining of dissected brains with anti-TH antibody, and ( $\mathbf{E}_{\mathbf{2}}$ ) quantification of DA PPL1 neurons at the indicated adult transgenic flies' brain. $\mathbf{F}$ Locomotion (climbing) activity of the shown transgenic flies. In (A-F) Tgs were expressed specifically in the nervous tissues (Gal4 ${ }^{\mathrm{TH}}$ and/or Gal4 ${ }^{\mathrm{Elav}}$ ). Gene expression in (A) was plotted vs. control set to $1 ; R^{2}$. $32 / r p 49$ gene was used as reference. Bars, $\pm S D ; n=3,{ }^{*} P<0.05 ;{ }^{* *} P<0.01$.

mitophagy rates in flies' tissues; furthermore, our data indicate that $\mathrm{cncC} / \mathrm{Nrf2}$ is a transcriptional regulator of park and Pink1 genes. Reportedly, a subset of the cytoplasmic Nrf2/Keap1 ROSsensing complexes are tethered to OMM via the interaction of Keap1 with the mitochondrial protein PGAM5 [54], while in C. elegans, Nrf2 coordinates both mitochondrial removal and biogenesis upon oxidative stress [55]. Interestingly, 1,4-diphenyl- 1,2,3-triazole (PMI), was found to mediate LC3 recruitment to mitochondria and to increase mitochondrial p62 co-localization in a Nrf2-dependent way [56]. Our data showed that cncC/Nrf2 can mediate mitophagy at the park or Pink1 KD genetic backgrounds in a $\operatorname{ref}(2) \mathrm{P} / \mathrm{p} 62$-independent manner, suggesting that Nrf2 likely engages additional ubiquitin receptors to mediate mitophagy. Indeed, Nrf2 is a transcriptional activator in mammalian cells of 
the autophagy receptor NDP52 [57]; yet the identification of Nrf2inducible receptors in Drosophila mitophagy should await further studies.

Finally, we observed that Nrf2 activation in the fly model largely rescued park or Pink1 KD-mediated neuromuscular degenerative phenotypes. Specifically, cncC/Nrf2-targeted activation in neuronal tissues expressing park or Pink1 RNAi, reduced oxidative load, tended to increase mitophagy and rescued loss of DA neurons in the brain. In DA neurons the park/Pink1 pathway is overactive, probably because of the high mitostatic stress that these cells face [58]; also, increased mitophagy rates due to cncC/Nrf2 OE in surrounding to DA neurons cells, could establish a by-stander effect that reduces oxidative load and provides survival signals. Consistently, Nrf2-activating molecules such as sulforaphane or RTA-40 can restore mitochondrial membrane potential and have protective effects in neurodegeneration [59]. Thus, Nrf2 activation can represent a promising therapeutic intervention in degenerative diseases.

\section{AVAILABILITY OF DATA AND MATERIALS}

The datasets generated and/or analyzed during the current study are available from the corresponding author on reasonable request.

\section{REFERENCES}

1. Gumeni S, Trougakos IP. Cross talk of proteostasis and mitostasis in cellular homeodynamics, ageing, and disease. Oxid Med Cell Longev. 2016;2016:4587691.

2. Ryan BJ, Hoek S, Fon EA, Wade-Martins R. Mitochondrial dysfunction and mitophagy in Parkinson's: from familial to sporadic disease. Trends Biochem Sci. 2015:40:200-10

3. Kerr JS, Adriaanse BA, Greig NH, Mattson MP, Cader MZ, Bohr VA, et al. Mitophagy and Alzheimer's disease: cellular and molecular mechanisms. Trends Neurosci. 2017;40:151-66.

4. Bose A, Beal MF. Mitochondrial dysfunction in Parkinson's disease. J Neurochem. 2016;1:216-31.

5. Anding AL, Baehrecke EH. Cleaning house: selective autophagy of organelles. Dev Cell. 2017:41:10-22.

6. Youle RJ, Narendra DP. Mechanisms of mitophagy. Nat Rev Mol Cell Biol. 2011;12:9-14.

7. Narendra D, Tanaka A, Suen DF, Youle RJ. Parkin is recruited selectively to impaired mitochondria and promotes their autophagy. J Cell Biol. 2008;183:795-803.

8. Kaushik S, Cuervo AM. The coming of age of chaperone-mediated autophagy. Nat Rev Mol Cell Biol. 2018;19:365-81.

9. Pickles S, Vigié P, Youle RJ. Mitophagy and quality control mechanisms in mitochondrial maintenance. Curr Biol. 2018;28:170-85.

10. Exner N, Lutz AK, Haass C, Winklhofer KF. Mitochondrial dysfunction in Parkinson' $s$ disease: molecular mechanisms and pathophysiological consequences. EMBO J. 2012;31:3038-62.

11. Valente EM, Abou-Sleiman PM, Caputo V, Muqit MM, Harvey K, Gispert S, et al. Hereditary early-onset Parkinson's disease caused by mutations in PINK1. Science. 2004;304:1158-60.

12. Fang EF, Hou $Y$, Palikaras $K$, Adriaanse BA, Kerr JS, Yang B, et al. Mitophagy inhibits amyloid- $\beta$ and tau pathology and reverses cognitive deficits in models of Alzheimer's disease. Nat Neurosci. 2019:22:401-12.

13. Holmström KM, Finkel T. Cellular mechanisms and physiological consequences of redox-dependent signalling. Nat Rev Mol Cell Biol. 2014;15:411-21.

14. Schieber $M$, Chandel NS. ROS function in redox signaling and oxidative stress. Curr Biol. 2014;24:453-62.

15. Cuadrado A, Rojo Al, Wells G, Hayes JD, Cousin SP, Rumsey WL, et al. Therapeutic targeting of the NRF2 and KEAP1 partnership in chronic diseases. Nat Rev Drug Discov. 2019;18:295-317.

16. Tsakiri EN, Trougakos IP. The amazing ubiquitin-proteasome system: structural components and implication in aging. Int Rev Cell Mol. 2015;314:171-237.

17. Rousseau A, Bertolotti A. An evolutionarily conserved pathway controls proteasome homeostasis. Nature. 2016:536:184-9.

18. Elkouzi A, Vedam-Mai V, Eisinger RS, Okun MS. Emerging therapies in Parkinson disease repurposed drugs and new approaches. Nat Rev Neurol. 2019;15:204-23.

19. Kaushik S, Cuervo AM. Proteostasis and aging. Nat Med. 2015;2:1406-15.
20. Holmström KM, Kostov RV, Dinkova-Kostova AT. The multifaceted role of Nrf2 in mitochondrial function. Curr Opin Toxicol. 2016;1:80-91.

21. Tsakiri EN, Gumeni S, lliaki KK, Benaki D, Vougas K, Sykiotis GP, et al. Hyperactivation of Nrf2 increases stress tolerance at the cost of aging acceleration due to metabolic deregulation. Aging Cell. 2019a;18:e12845.

22. Gumeni S, Evangelakou Z, Tsakiri EN, Scorrano L, Trougakos IP. Functional wiring of proteostatic and mitostatic modules ensures transient organismal survival during imbalanced mitochondrial dynamics. Redox Biol. 2019;24:101219.

23. Lionaki E, Markaki M, Palikaras K, Tavernarakis N. Mitochondria, autophagy and age-associated neurodegenerative diseases: new insights into a complex interplay. Biochim Biophys Acta. 2015;1847:1412-23.

24. Geisler S, Holmström KM, Skujat D, Fiesel FC, Rothfuss OC, Kahle PJ, et al. PINK1/ Parkin-mediated mitophagy is dependent on VDAC1 and p62/SQSTM1. Nat Cell Biol. 2010;12:119-31.

25. Komatsu M, Kurokawa H, Waguri S, Taguchi K, Kobayashi A, Ichimura $\mathrm{Y}$, et al. The selective autophagy substrate p62 activates the stress responsive transcription factor Nrf2 through inactivation of Keap1. Nat Cell Biol. 2010;12:213-23.

26. Tsakiri EN, Sykiotis GP, Papassideri IS, Gorgoulis VG, Bohmann D, Trougakos IP. Differential regulation of proteasome functionality in reproductive vs. somatic tissues of Drosophila during aging or oxidative stress. FASEB J. 2013;27:407-20.

27. Tsakiri EN, Gumeni S, Vougas K, Pendin D, Papassideri I, Daga A, et al. Proteasome dysfunction induces excessive proteome instability and loss of mitostasis that can be mitigated by enhancing mitochondrial fusion or autophagy. Autophagy. 2019b;15:1757-73.

28. Park J, Lee SB, Lee S, Kim Y, Song S, Kim S, et al. Mitochondrial dysfunction in Drosophila PINK1 mutants is complemented by parkin. Nature. 2006:441:1157-61.

29. Nagoshi E. Drosophila models of sporadic Parkinson's disease. J Mol Sci. 2018;19:3343.

30. Lee JJ, Sanchez-Martinez A, Martinez Zarate A, Benincá C, Mayor U, Clague MJ, et al. Basal mitophagy is widespread in Drosophila but minimally affected by loss of Pink1 or parkin. J Cell Biol. 2018;217:1613-22.

31. Corona JC, Duchen MR. PPARY and PGC-1a as therapeutic targets in Parkinson's. Neurochem Res. 2015;40:308-16.

32. López-Otín C, Blasco MA, Partridge L, Serrano M, Kroemer G. The hallmarks of aging. Cell. 2013;153:1194-217.

33. Lehmann G, Udasin RG, Ciechanover A. On the linkage between the ubiquitinproteasome system and the mitochondria. Biochem Biophys Res Commun. 2016;473:80-6

34. Doherty J, Baehrecke EH. Life, death and autophagy. Nat Cell Biol. 2018;20:1110-7.

35. Dagda RK, Cherra SJ 3rd, Kulich SM, Tandon A, Park D, Chu CT, et al. Loss of PINK1 function promotes mitophagy through effects on oxidative stress and mitochondrial fission. J Biol Chem. 2009;284:13843-55.

36. Yamano K, Youle RJ. PINK1 is degraded through the $\mathrm{N}$-end rule pathway. Autophagy. 2013;9:1758-69.

37. Iguchi $M$, Kujuro $Y$, Okatsu $K$, Koyano $F$, Kosako $H$, Kimura $M$, et al. Parkincatalyzed ubiquitin-ester transfer is triggered by PINK1-dependent phosphorylation. J Biol Chem. 2013;288:22019-32.

38. Tanaka A, Cleland MM, Xu S, Narendra DP, Suen DF, Karbowski M, et al. Proteasome and p97 mediate mitophagy and degradation of mitofusins induced by Parkin. J Cell Biol. 2010;191:1367-80.

39. Chan NC, Salazar AM, Pham AH, Sweredoski MJ, Kolawa NJ, Graham RL, et al. Broad activation of the ubiquitin-proteasome system by Parkin is critical for mitophagy. Hum Mol Genet. 2011;20:1726-37.

40. Liu W, Vives-Bauza C, Acín-Peréz- R, Yamamoto A, Tan Y, Li Y, et al. PINK1 defect causes mitochondrial dysfunction, proteasomal deficit and alpha-synuclein aggregation in cell culture models of Parkinson's disease. PLoS ONE. 2009;4: e4597.

41. Boland B, Yu WH, Corti O, Mollereau B, Henriques A, Bezard E, et al. Promoting the clearance of neurotoxic proteins in neurodegenerative disorders of ageing. Nat Rev Drug Discov. 2018;17:660-88

42. Gómez-Sánchez R, Yakhine-Diop SM, Bravo-San Pedro JM, Pizarro-Estrella $E$ Rodríguez-Arribas M, Climent V, et al. PINK1 deficiency enhances autophagy and mitophagy induction. Mol Cell Oncol. 2015;3:e1046579.

43. Kanki T, Klionsky DJ. Mitophagy in yeast occurs through a selective mechanism. J Biol Chem. 2008;283:32386-93.

44. Kamienieva I, Duszyński J, Szczepanowska J. Multitasking guardian of mitochondrial quality: parkin function and Parkinson's disease. Transl Neurodegener. 2021;10:5.

45. Voigt A, Berlemann LA, Winklhofer KF. The mitochondrial kinase PINK1: functions beyond mitophagy. J Neurochem. 2016;139:232-9.

46. Palikaras K, Lionaki E, Tavernarakis N. Mechanisms of mitophagy in cellular homeostasis, physiology and pathology. Nat Cell Biol. 2018;20:1013-22.

47. Demontis F, Perrimon N. FOXO/4E-BP signaling in Drosophila muscles regulates organism-wide proteostasis during aging. Cell. 2010;143:813-25. 
48. Neufeld TP. Genetic manipulation and monitoring of autophagy in Drosophila. Methods Enzymol. 2008;451:653-67.

49. McWilliams TG, Prescott AR, Montava-Garriga L, Ball G, Singh F, Barini E, et al. Basal mitophagy occurs independently of PINK1 in mouse tissues of high metabolic demand. Cell Metab. 2018;27:439-49.

50. Durcan TM, Fon EA. The three 'P's of mitophagy: PARKIN, PINK1, and posttranslational modifications. Genes Dev. 2015;29:989-99.

51. Lazarou M, Jin SM, Kane LA, Youle RJ. Role of PINK1 binding to the TOM complex and alternate intracellular membranes in recruitment and activation of the E3 ligase Parkin. Dev Cell. 2012;22:320-33.

52. Strappazzon F, Nazio F, Corrado M, Cianfanelli V, Romagnoli A, Fimia GM, et al. AMBRA1 is able to induce mitophagy via LC3 binding, regardless of PARKIN and p62/SQSTM1. Cell Death Differ. 2015;22:419-32.

53. Chen G, Kroemer G, Kepp O. Mitophagy: an emerging role in aging and ageassociated diseases. Front Cell Dev Biol. 2020;8:200.

54. Lo SC, Hannink M. PGAM5 tethers a ternary complex containing Keap1 and Nrf2 to mitochondria. Exp Cell Res. 2008;314:1789-803.

55. Palikaras K, Lionaki E, Tavernarakis N. Balancing mitochondrial biogenesis and mitophagy to maintain energy metabolism homeostasis. Cell Death Differ. 2015;22:1399-401.

56. East DA, Fagiani F, Crosby J, Georgakopoulos ND, Bertrand H, Schaap M, et al. PMI: a $\Delta \Psi \mathrm{m}$ independent pharmacological regulator of mitophagy. Chem Biol. 2014;21:1585-96.

57. Pajares M, Cuadrado A, Rojo Al. Modulation of proteostasis by transcription factor NRF2 and impact in neurodegenerative diseases. Redox Biol. 2017;11:543-53.

58. Pickrell AM, Youle RJ. The roles of PINK1, parkin, and mitochondrial fidelity in Parkinson's disease. Neuron. 2015;85:257-73.

59. Dinkova-Kostova AT, Abramov AY. The emerging role of Nrf2 in mitochondrial function. Free Radic Biol Med. 2015;88:179-88.

\section{ACKNOWLEDGEMENTS}

We thank Dirk Bohmann (University of Rochester, NY, USA), Alexander Whitworth (University of Cambridge, UK) and Genny Orso (University of Padova) for fly lines. SG and MSM are recipients of a Post-Doc and PhD, respectively, fellowship from the Hellenic State Scholarships Foundation; this research is co-financed by Greece and the European Union (European Social Fund through the Operational Programme "Human Resources Development, Education and Lifelong Learning 2014-2020"). I.P.T. acknowledges funding the Hellenic GSRT project BIOIMAGINGGR (MIS 5002755).

\section{AUTHOR CONTRIBUTIONS}

I.P.T. designed and supervised the study; S.G., E.D.P., M.S.M., and I.P.T. conducted experiments or interpreted the data; S.G., E.D.P., and I.P.T. wrote the manuscript.

\section{COMPETING INTERESTS}

The authors declare no competing interests.

Ethics

The study did not require ethical approval.

\section{ADDITIONAL INFORMATION}

Supplementary information The online version contains supplementary material available at https://doi.org/10.1038/s41419-021-03952-w.

Correspondence and requests for materials should be addressed to I.P.T.

Reprints and permission information is available at http://www.nature.com/ reprints

Publisher's note Springer Nature remains neutral with regard to jurisdictional claims in published maps and institutional affiliations.

(C)

Open Access This article is licensed under a Creative Commons Attribution 4.0 International License, which permits use, sharing, adaptation, distribution and reproduction in any medium or format, as long as you give appropriate credit to the original author(s) and the source, provide a link to the Creative Commons license, and indicate if changes were made. The images or other third party material in this article are included in the article's Creative Commons license, unless indicated otherwise in a credit line to the material. If material is not included in the article's Creative Commons license and your intended use is not permitted by statutory regulation or exceeds the permitted use, you will need to obtain permission directly from the copyright holder. To view a copy of this license, visit http://creativecommons. org/licenses/by/4.0/.

(c) The Author(s) 2021 